\title{
CONNECTIVITY SEARCHLIGHT: A NOVEL APPROACH FOR MRI INFORMATION MAPPING USING MULTIVARIATE CONNECTIVITY
}

\author{
Soheil Faridi ${ }^{1,2}$, Jonas Richiardi ${ }^{3,4}$, Patrik Vuilleumier ${ }^{3}$, and Dimitri Van De Ville Vi,2 $^{1,2}$ \\ ${ }^{1}$ Institute of Bioengineering, Federal Institute of Technology of Lausanne, CH-1015 Lausanne \\ ${ }^{2}$ Medical Image Processing Lab, University of Geneva, CH-1211 Geneva \\ ${ }^{3}$ Laboratory of Neurology and Imaging of Cognition, University of Geneva, CH-1211 Geneva \\ ${ }^{4}$ Functional Imaging in Neuropsychiatric Disorders Lab, Stanford University, USA
}

\begin{abstract}
Brain mapping using magnetic resonance imaging (MRI) is traditionally performed using voxel-wise statistical hypothesis testing. Such mass-univariate approach ignores subtle spatial interactions. The searchlight method, in contrast, uses a multivariate predictive model in each local neighborhood in brain space-named the searchlight. The classification performance is then reported at the center of the searchlight to build an information map. We extend the searchlight technique to take into account additional voxels that can be considered as a meaningful network; i.e., we define a criterion of multivariate connectivity to identify voxels that are statistically dependent on those in searchlight. We coin the term "connectivity searchlight" for the extended searchlight. Using simulated data, we empirically show improved performance for brain regions with low signal-to-noise ratio and recovery of underlying network structures that would otherwise remain hidden. The proposed methodology is general and can be applied to both functional and structural data. We also demonstrate promising results on a well-known fMRI dataset where images of different categories are presented.
\end{abstract}

Index Terms- Magnetic resonance imaging, pattern recognition, multivariate analysis, functional connectivity

\section{INTRODUCTION}

Magnetic resonance imaging (MRI) has opened unprecedented ways to explore brain structure and function noninvasively. Traditionally, univariate statistical methods are used to analyze the data voxel-by-voxel. However, it has been shown that distributed patterns can carry a lot of information, such as about different image categories [1]. Recent advances have applied tools from machine learning to perform multivariate pattern analysis (MVPA), commonly termed "brain

This work was supported in part by the Swiss National Science Foundation (under grant PP00P2-123438) and in part by the Center for Biomedical Imaging (CIBM), and in part by the Société Académique de Genève and the FOREMANE foundation. decoding" or "mind reading", as the condition is inferred from the data $[2,3]$.

One elegant technique for brain decoding is the so-called searchlight method [4]. For each voxel, a local neighborhood is considered (e.g., sphere or cube) and these voxels' values are used for classification or regression. The performance is then reported at the center of the searchlight to build an information map; i.e., it reflects the amount of information that is available in the searchlight to predict the condition. This way, subtle local interactions between the voxels can be exploited successfully. However, since the searchlight is local by construction, it heavily relies on the principle of functional segregation; i.e., local processing and specialization of the brain.

Recently, there has been increasing interest of the community for functional and structural connectivity [5], and networks derived from such measures. When combined with pattern recognition, it has been shown that functional connectivity between atlas-based regions-of-interest (ROIs) can decode cognitive state $[6,7]$ and patient status [8]. This motivated us to look for an extension of the searchlight method such that information can be decoded from a network of which the searchlight is part. To the best of our knowledge, such an extension has not been proposed before. Only in recent work, voxels in a limited set of ROIs where combined to decode sensory-to-motor mapping based on connectivity between the ROIs [9].

We propose two new elements to extend the original searchlight. First, we define a criterion of connectivity to identify voxels in the brain that are statistically dependent on the searchlight; our criterion is multivariate and goes beyond traditional correlation measures to quantify (functional) connectivity ${ }^{1}$. Since MRI data can have global confounds (e.g., motion in functional MRI), we also provide the possibility to include nuisance factors that can explain background signal. We then create the "connectivity searchlight" by extending the searchlight with connected voxels, defining a network related to the searchlight that is fed into the classifier. To show

\footnotetext{
${ }^{1}$ We do not discuss effective connectivity here, which can reveal causal relationships based on predefined models and richer data [5].
} 
the potential of this method, we demonstrate its feasibility on both simulated and experimental MRI data, and compare it against the conventional searchlight.

\section{SEARCHLIGHT FOLLOWS CONNECTIVITY}

\subsection{Conventional Searchlight Approach}

Let us introduce the data structure. We are given $K$ brain scans, which could be functional scans for stimulus-induced activity or structural scans for multiple subjects. Each volumetric scan has a size of $V_{1} \times V_{2} \times V_{3}$ voxels, which can be collapsed into a vector of size $V=V_{1} V_{2} V_{3}$. The full data matrix $\mathbf{D}$ has then size $K \times V$, where the scans have been arranged row-wise. We also have a vector $\mathbf{y} \in \mathbb{N}^{K \times 1}$ that contains the condition of each scan. The searchlight approach is then using a 3-D sliding window that contains a local neighborhood $\mathcal{S}_{i}$ centered around each voxel $i$. The shape and size of the searchlight are parameters to be defined; e.g., we can use the commonly deployed cubic searchlight which includes $L=(2 m+1)^{3}=\left|\mathcal{S}_{i}\right|$ voxels when $m$ is the number of neighbors is each dimension. We denote $\mathbf{D}_{\mathcal{S}_{i}}$ the subset of voxels in the data matrix that are included in the searchlight. The purpose is to predict the conditions of the scans $\mathbf{y}$ by using the searchlight voxels' values as features. One can then build an "information map" by reporting the performance of each searchlight $\mathcal{S}_{i}$ in the center voxel $i$ [4]. Since this is done in within the cross-validation framework, the data matrix is split each time into $\mathbf{D}_{\text {train }, \mathcal{S}_{i}}\left(K^{\prime} \times L\right)$ and $\mathbf{D}_{\text {test }, \mathcal{S}_{i}}\left(K^{\prime \prime} \times L\right)$.

\subsection{Multivariate Connectivity}

The searchlight is using only information from the local neighborhood $\mathcal{S}_{i}$, thus exploiting only local specialization of the brain. However, the brain is also a globally integrated system, which means that distant brain regions operate in brain networks. To extend the searchlight with "connected" voxels, we propose the following criterion for multivariate connectivity. Notice that connectivity can be both functional (e.g., timecourses are correlated) or morphometric (e.g., different anatomical features varies similarly in subjects).

Let us consider the searchlight $\mathcal{S}_{i}$. We define multivariate connectivity between the searchlight and other voxels as the ability of searchlight data to explain non-searchlight voxels. More specifically, we create a general linear model (GLM) that consists of the searchlight data $\mathbf{D}_{\text {train, } \mathcal{S}_{i}}$ as regressors to explain the signal at any other voxel $j$. Since MR data is often contaminated by strong background signals, we also add nuisance regressors that are extracted as the first $L_{0}$ principal components $\mathbf{U}_{L_{0}}$ of the full data matrix $\mathbf{D}_{\text {train }}$. The $K^{\prime} \times(L+$ $L_{0}$ ) design matrix for voxel $i$ then becomes

$$
\mathbf{X}_{i}=\left[\mathbf{D}_{\text {train }, \mathcal{S}_{i}} \mid \mathbf{U}_{L_{0}}\right]
$$

which leads to the following GLM:

$$
\mathbf{d}^{(j)}=\mathbf{X}_{i} \beta_{i}^{(j)}+\mathbf{n}_{i}
$$

where $\beta_{i}^{(j)}$ is the $\left(L+L_{0}\right) \times 1$ parameter vector of the searchlight centered at voxel $i$ to explain data at $j$, and $\mathbf{n}_{i}$ is assumed additive zero-mean and independent Gaussian noise. Using the ordinary least-squares solution, we can find the estimate and the residuals as

$$
\begin{aligned}
& \hat{\mathbf{d}}_{i}^{(j)}=\mathbf{X}_{i} \underbrace{\left(\mathbf{X}_{i}^{T} \mathbf{X}_{i}\right)^{-1} \mathbf{X}_{i}^{T} \mathbf{d}^{(j)}}_{\boldsymbol{\beta}_{i}^{(j)}}, \\
& \hat{\mathbf{e}}_{i}^{(j)}=\mathbf{d}^{(j)}-\hat{\mathbf{d}}_{i}^{(j)} .
\end{aligned}
$$

To test the significance of the explained signal by the GLM, we compute the $t$-statistic for the contrast

$$
\sum_{l=1}^{L} \beta_{i}^{(j)}[l]=\underbrace{\beta_{i}^{(j)}}_{\underbrace{[\overbrace{11 \ldots 1}^{L \text { times }}}_{\text {contrast vector } \mathbf{c}^{T}} \overbrace{00 \ldots 0}^{L_{0} \text { times }}}
$$

as

$$
t_{i}^{(j)}=\frac{\mathbf{c}^{T} \boldsymbol{\beta}_{i}^{(j)}}{\sqrt{\frac{\mathbf{c}^{T}\left(\mathbf{X}_{i}^{T} \mathbf{X}_{i}\right)^{-1} \mathbf{c} \hat{\mathbf{e}}_{i}^{(j), T} \hat{\mathbf{e}}_{i}^{(j)}}{K^{\prime}-L-L_{0}}}},
$$

which follows a Student t-distribution with $K^{\prime}-L-L_{0}$ degrees-of-freedom under the null hypothesis $\mathcal{H}_{0}: E\left[\mathbf{c}^{T} \boldsymbol{\beta}_{i}^{(j)}\right]=$ 0 . We define the set of voxels $\mathcal{C}_{i}$ connected with the searchlight $\mathcal{S}_{i}$ as those that survive the thresholding $\left|t_{i}^{(j)}\right|>T$, where $T$ is the threshold for $\alpha$-level $0.05 /(V-L)$, which is Bonferroni corrected for the number of voxels to be tested.

\subsection{Extending the Searchlight}

Based on the outcome of the multivariate connectivity criterion, we construct the "connectivity searchlight" as the union $\mathcal{S}_{i}^{\prime}=\mathcal{S}_{i} \cup \mathcal{C}_{i}$. In Fig. 1, we illustrate the principle of extending the searchlight with connected voxels. Given the feature vectors $\mathbf{D}_{\text {train, } \mathcal{S}_{i}^{\prime}}$, we learn a support vector classifier (SVC) that should predict $\mathbf{y}_{\text {train }}$. The performance on the test data $\mathbf{D}_{\text {test }, \mathcal{S}_{i}^{\prime}}$ is then reported in the information map for the central voxel of each searchlight.

\section{RESULTS}

\subsection{Simulated Data}

We simulated a noisy dataset with known underlying "networks" of simultaneously active regions for two different conditions. In particular, our dataset consists of volumes of $64 \times$ $52 \times 3$ voxels. In Fig. 2 a, we show the labeled ground truth for the central slice; all three slices are identical. In the first condition, the left eye, upper lip, and the nose are "active". In the second condition, the right eye, lower lip, and the same 


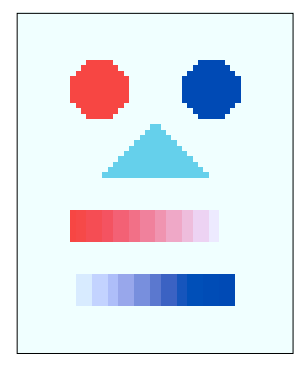

(a)

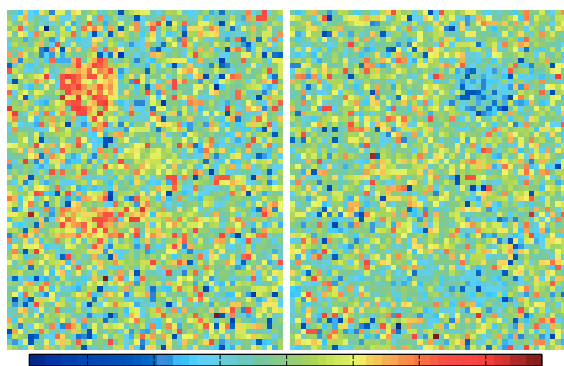

(b)

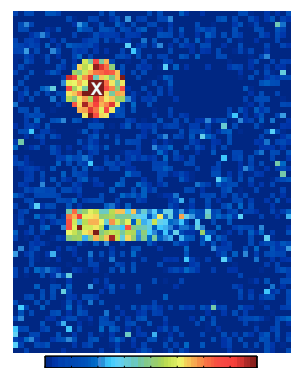

(c)

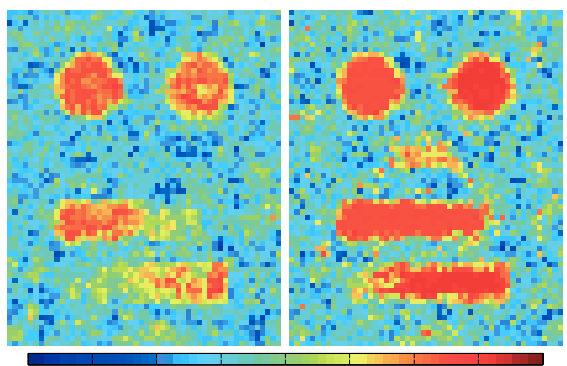

(d)

Fig. 2: (a) The ground truth of the synthetic data where red and blue regions carry the signal for the two conditions, respectively; while the cyan region has the signal for both. (b) Two example trials for each condition. (c) $t$-map for the searchlight positioned in the left eye (indicated by the crosshair). (d) Information maps for searchlight and connectivity searchlight, respectively.

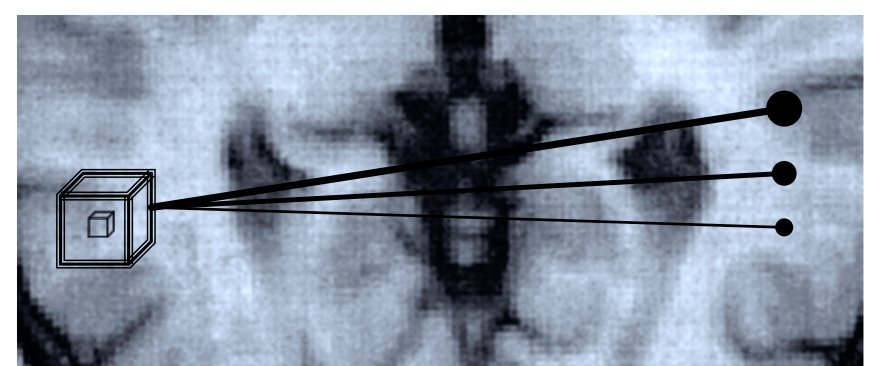

Fig. 1: Principle of connectivity searchlight. The cube represents the initial searchlight around the central voxel. Using regression, we extend the searchlight with other voxels that have similar signal content.

nose are active. There is a gradient of weak to strong activity on the lips. The volumes $I_{1}(\mathbf{x})$ and $I_{2}(\mathbf{x})$ indicate the activity strength for both conditions, respectively. We then generate $K=100$ trials as

$$
\begin{gathered}
\mathbf{y}=\overbrace{11 \ldots 1}^{K / 2 \text { times }} \overbrace{22 \ldots 2}^{K / 2}]^{T} \text { times } \\
\mathbf{D}_{j, i}=\sum_{k=1}^{2} \delta_{\left(y_{j}-k\right)} I_{k}\left(\mathbf{x}_{i}\right)\left(b_{k}+n_{k}\left(j ; \sigma_{s}\right)\right)+n_{s}\left(j ; \sigma_{n}\right),
\end{gathered}
$$

where $\delta$ is the Kronecker delta, $b_{k}$ is the baseline signal (i.e., $\left.b_{1}=1, b_{2}=-1\right)$, and $n(\cdot ; \sigma)$ are Gaussian noise variables with zero mean and standard deviation $\sigma$; we have chosen $\sigma_{s}=\sigma_{1}=\sigma_{2}=0.8$. Notice that the signal variations $b_{k}+n_{k}$ for condition $k$ also contain "randomness", which is consistent in the corresponding regions $I_{k}$ though. In Figs. 2b, we show two representative scans for the two conditions. We selected the searchlight to be a $3 \times 3 \times 3$ cube, we only process the central slice for easier visualization. The training set $\mathbf{D}_{\text {train }}$ contains half of the trials (i.e., 25) for each condition. In Fig. 2c, we show the statistical map of $t$-values for multivariate connectivity when the searchlight is positioned in the middle of the left eye. Clearly, the "network" of regions with similar activity for condition 1 are revealed with high significance (i.e., threshold $T=4.4$ Bonferronicorrected at $5 \%$ ). The connectivity searchlight includes the data from all voxels that survive this thresholding procedure. In Figs. 2d, we show the information maps obtained when using the conventional searchlight and connectivity searchlight, respectively. In particular, we report the classification performance at the center of each searchlight. The conventional searchlight obtains satisfactory performance from local neighborhoods with strong signal-to-noise ratio (e.g., the eyes and the high-activity parts of the mouths). The connectivity searchlight is able to improve on these results by (1) better classification performance in many regions; (2) including considerably more regions with low signal (e.g. low-activity parts of lips); (3) including regions that are connected (i.e., same activity variations), but that are not informative for the classification, such as the nose.

\subsection{Experimental fMRI Data}

We also tested our method on a subset of the well-known Haxby dataset [1]. In particular, we took data for one subject where images of houses and faces were shown, in total 216 scans are available. The original volumes have size $53 \times 63 \times 46$, but we limited ourselves to the central axial slices 22,23 , and 24 . The data was split in half between training and testing. The same cubic searchlight as before was used. In Fig. 3a, we show the t-maps for two different positions of the searchlight (indicated with by the blue crosshair). On the left, the searchlight is located in the auditory cortex, which is not an informative region for the task at hand. The connectivity searchlight only slightly extends its spatial neighborhood. On the right, the searchlight is located in the anterior cingulate cortex, which is a high-level cognitive region. The connectivity searchlight now includes the core of the caudate and parts of visual cortex in the occipital lobe. In Fig. 3b, the classification performance is reported for the central voxel of the searchlight. On the left, as expected the conventional searchlight is most informative in the visual cortex. On the right, the connectivity searchlight performs very well for different brain regions, including a larger part of the visual cortex, left insula, and anterior cingulate cortex. The (spatial) average classification performance is $66 \%$ and $86 \%$, respectively. 


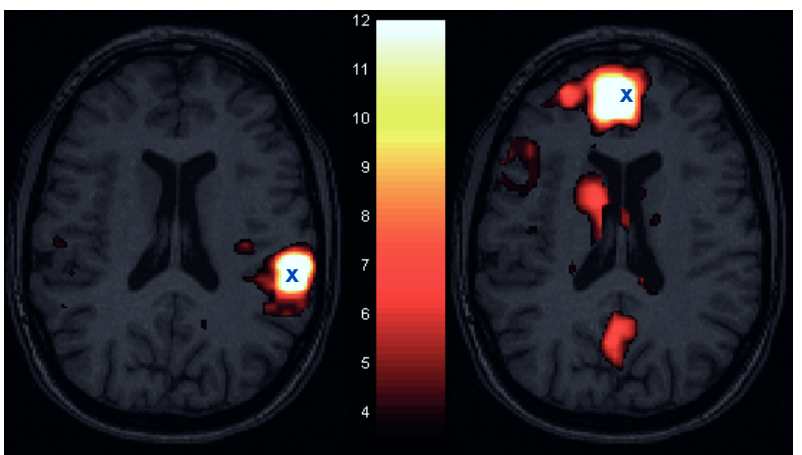

(a)

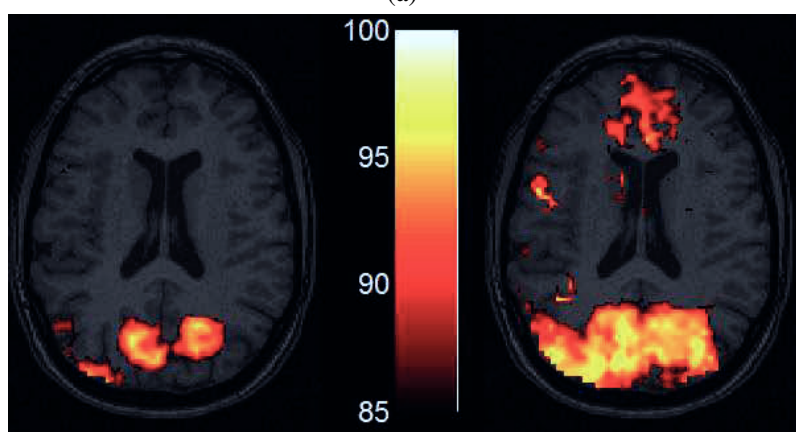

(b)

Fig. 3: Results for the Haxby dataset (faces versus houses). (a) Connectivity map (t-value) for two different locations of the searchlight indicated by the blue crosshairs. (b) Comparison between information maps for conventional searchlight (left) and connectivity searchlight (right).

\section{DISCUSSION}

The searchlight is a powerful technique that allows exploiting multivariate information locally. The main contribution of our approach is to extend the searchlight with regions that form a network according to our criterion of multivariate connectivity; i.e., voxels in the searchlight that explain the signal elsewhere with high significance.

As the connectivity searchlight contains more voxels than the conventional searchlight, it is not surprising that classification performance is improved since more voxels with high signal-to-noise are included. However, it is important to note that the connectivity criterion is not necessarily related to discriminative features. Such an example was shown in the nose region of the simulated dataset: since the signal was (equally) shared for both conditions, these voxels cannot contribute successfully to the classification task as they carry no discriminative information. Still they are included in the connectivity searchlight as they are part of the network for each condition and it might be important for the neuroscientist to have a picture of the full network at work. In fact, the main goal of our method is to reveal network structures in a more meaningful way.

The shape and size of the searchlight are parameters that need to be selected. Based on preliminary experiments, we observed that the multivariate connectivity criterion locally extends searchlight with "important" neighboring voxels, thus the settings of these parameters might be less critical.

Controlling the influence of global confounds is an important issue that needs to be dealt with, especially for functional data. Here we propose to include nuisance covariates in the GLM from whole-brain principal component analysis. There is an interesting link with sparse multivariate autoregression models to build information flow models of the brain $[10,11]$. We plan to deepen this link in future work.

\section{CONCLUSION}

We have introduced a connectivity-based extension of the searchlight method, which can be applied to both functional and structural MRI data. Preliminary results on simulated and real fMRI data demonstrate the feasibility of the approach and its potential to reveal networks of the brain.

\section{REFERENCES}

[1] J. V. Haxby, M. I. Gobbini, M. L. Furey, A. Ishai, J. L. Schouten, and P. Pietrini, "Distributed and overlapping representations of faces and objects in ventral temporal cortex," Science, vol. 293, no. 5539, pp. 2425-2430, 2001.

[2] Y. Kamitani and F. Tong, "Decoding the visual and subjective contents of the human brain," Nature Neuroscience, vol. 8 , no. 5, pp. 679-685, 2005.

[3] T. M. Mitchell, S. V. Shinkareva, A. Carlson, K. Chang, V. L. Malave, R. A. Mason, and M. A. Just, "Predicting human brain activity associated with the meanings of nouns," Science, vol. 320, no. 5880, pp. 1191-1195, 2008.

[4] N. Kriegeskorte, R. Goebel, and P. Bandettini, "Informationbased functional brain mapping," Proceedings of the National Academy of Sciences of the United States of America, vol. 103, no. 10, pp. 3863-3868, Mar. 2006.

[5] K. J. Friston, "Functional and effective connectivity: a review," Brain Connectivity, vol. 1, no. 1, pp. 13-36, 2011.

[6] J. Richiardi, H. Eryilmaz, S. Schwartz, P. Vuilleumier, and D. Van De Ville, "Decoding brain states from fMRI connectivity graphs," NeuroImage, vol. 56, no. 2, pp. 616-626, May 2011.

[7] W. R. Shirer, S. Ryali, E. Rykhlevskaia, V. Menon, and M. D. Greicius, "Decoding subject-driven cognitive states with whole-brain connectivity patterns," Cerebral cortex, vol. 22, no. 1, pp. 158-165, Jan. 2012.

[8] K. Wang, T. Jiang, M. Liang, L. Wang, L. Tian, X. Zhang, $\mathrm{K}$. Li, and Z. Liu, "Discriminative analysis of early Alzheimer's disease based on two intrinsically anti-correlated networks with resting-state fMRI," MICCAI, vol. 9, no. Pt 2, pp. 340-347, 2006.

[9] J. Heinzle, M. A. Wenzel, and J.-D. Haynes, "Visuomotor functional network topology predicts upcoming tasks," The Journal of Neuroscience, vol. 32, no. 29, pp. 9960-9968, 2012.

[10] P. A. Valdés-Sosa, J. M. Sánchez-Bornot, A. Lage-Castellanos, M. Vega-Hernández, J. Bosch-Bayard, L. Melie-García, and E. Canales-Rodríguez, "Estimating brain functional connectivity with sparse multivariate autoregression," Philosophical transactions of the Royal Society of London. Series B, Biological sciences, vol. 360, no. 1457, pp. 969-981, May 2005.

[11] R. Garg, G. A. Cecchi, and A. R. Rao, "Full-brain autoregressive modeling (farm) using fMRI," NeuroImage, vol. 58, no. 2, pp. 416-441, Sep. 2011. 